\title{
Diagnosis of Alzheimer's Disease Using Brain Network
}

\author{
Ramesh Kumar Lama and Goo-Rak Kwon* \\ The Alzheimer's Disease Neuroimaging Initiative, Department of Information and Communication Engineering, Chosun \\ University, Gwangju, South Korea
}

Recent studies suggest the brain functional connectivity impairment is the early event occurred in case of Alzheimer's disease (AD) as well as mild cognitive impairment (MCl). We model the brain as a graph based network to study these impairment. In this paper, we present a new diagnosis approach using graph theory based features from functional magnetic resonance ( $\mathrm{fMR}$ ) images to discriminate $\mathrm{AD}, \mathrm{MCl}$, and healthy control $(\mathrm{HC})$ subjects using different classification techniques. These techniques include linear support vector machine (LSVM), and regularized extreme learning machine (RELM). We used pairwise Pearson's correlation-based functional connectivity to construct the brain network. We compare the classification performance of brain network using Alzheimer's disease neuroimaging initiative (ADNI) datasets. Node2vec graph embedding approach is employed to convert graph features to feature vectors. Experimental results show that the SVM with LASSO feature selection method generates better classification accuracy

\section{OPEN ACCESS}

Edited by:

Szilvia Anett Nagy,

University of Pécs, Hungary

Reviewed by:

Kewei Chen,

Banner Alzheimer's Institute,

United States

Alexandra L. Young,

University College London,

United Kingdom

*Correspondence:

Goo-Rak Kwon

grkwon@chosun.ac.kr

Specialty section:

This article was submitted to

Brain Imaging Methods,

a section of the journal

Frontiers in Neuroscience

Received: 11 September 2020

Accepted: 06 January 2021

Published: 05 February 2021

Citation:

Lama RK and Kwon G-R (2021)

Diagnosis of Alzheimer's Disease

Using Brain Network.

Front. Neurosci. 15:605115. doi: 10.3389/fnins.2021.605115 compared to other classification technique.

Keywords: Alzhieimer's disease, brain network, node2vec, extreme learning machine, support vector machine

\section{INTRODUCTION}

Alzheimer's disease $(\mathrm{AD})$, which causes majority of dementia is a progressive neurodegenerative disease (American Psychiatric Association, 1994; Liu F. et al., 2014; Schmitter et al., 2015; Alzheimer's association, 2016). The subtle AD neuropathological process begins years before the visible progressive cognitive impairment, which is trouble to remember and learn new information. Currently there is no cure and treatment to slow or stop its progression. Currently, more research works are focused toward earlier intervention of AD. Thus accurate diagnosis of disease at its early stage makes great significance in such scenario.

With the availability of recent neuroimaging technology, promising result is obtained in the early and accurate detection of AD (Hanyu et al., 2010; Górriz et al., 2011; Gray et al., 2012). The study of progression of disease and early detection is carried out by using different imaging models, such as electroencephalography (EEG) (Pfefferbaum et al., 2000), functional magnetic resonance imaging (fMRI) (Masliah et al., 1993), single-photon emission computed tomography (SPECT) (Chen et al., 2013) and positron emission tomography (PET) (Ly et al., 2014).

Similarly, structural magnetic resonance imaging (MRI) (Hanyu et al., 1999; Canu et al., 2010; Bendlin et al., 2012) is the most commonly used imaging system for study of AD. The feature extracted from MRI is typically gray matter volumes and measured as important biomarker for the study of neurodegeneration, alterations of hippocampal white matter pathways is often observed in AD (Delbeuck et al., 2003; Liu Y. et al., 2014). Several studies reveal the alterations in widely distributed functional and structural connectivity pairs are prevalent in $\mathrm{AD}$ and mild cognitive impairment (MCI) (Delbeuck et al., 2007; Acosta-Cabronero et al., 2012). Additionally, in recent studies, the resting-state functional magnetic resonance imaging (rs-fMRI) has been widely used 
for the investigations of progression of AD (Stam et al., 2006; Sorg et al., 2007; Supekar et al., 2008; Doan et al., 2017). This imaging system evaluates the impulsive variabilities seen in the blood oxygenation level-dependent (BOLD) indications in various regions of the brain. Several studies are carried out based on aberrant regional spontaneous fluctuation of BOLD, functional connectivity and alteration in functional brain network. These studies are carried out in different networks, such as default mode network, somatomotor network, dorsal attention network, limbic network, and frontoparietal control network (Bullmore and Sporns, 2009). Thus, the graph theory based network analyses of human brain functional connectomes, provides better insights of the network structure to reveal abnormal patterns of organization of functional connectivity in $\mathrm{AD}$ infected brain (Rubinov and Sporns, 2010; Sporns, 2011).

Graph theory is a mathematical approach to study complex networks. Network is constructed of vertices which are interconnected by edges. Vertices in our case are brain regions. Graph theory is widely used as tool for identifying anatomically localized subnetworks associated with neuronal alterations in different neurodegenerative diseases (Bajo et al., 2010). In fMRI images, graph represents causal relations or correlations of different nodes in constructed networks. However, the brain network built by graph has non-Euclidian characteristics. Thus, applying machine learning techniques to analyze the brain networks is challenging. We use graph embedding to transform graphs to a vector or set of vectors to overcome this problem. Embedding captures the graph topology, vertexvertex relationship, and other relevant graph information. In the current study, we used node2vec graph embedding technique to transform vertex and edge of brain network graph to feature vector. With the help of this model we have analyzed and classified the networks of brain from fMRI data into $\mathrm{AD}, \mathrm{MCI}$, and HC.

\section{MATERIALS FOR THE STUDY}

\section{fMRI Dataset}

In our study, we have used the dataset from Alzheimer's disease neuroimaging initiative database $(\mathrm{ADNI})^{1}$. The ADNI database was launched in 2004. The database consists of subjects of age ranging from $55-90$ years. The goal of ADNI is to study the progression of the disease using different biomarkers. This includes clinical measures and assesses of the structures and functions of brain for the course of different disease states.

All participants were scanned using 3.0-Telsa Philips Achieva scanners at different centers. Same scanning protocol were followed for all participants and the set parameters were ratio of Repetition Time (TR) to Echo Time (TE) i.e., TR/TE $=3000 / 30 \mathrm{~ms}, 140$ volumes, also voxel thickness as $3.3 \mathrm{~mm}$, acquisition matrix size $=64 \times 64,48$ slices, flip angle $=80^{\circ}$ Similarly, 3D T1-weighted images were collected using MPRAGE sense2 sequences with acquisition type $3 \mathrm{D}$, field strength $=3$ Tesla, flip angle

${ }^{1}$ http://adni.loni.usc.edu/
9.0 degree, pixel spacing $\mathrm{X}=1.0547000169754028 \mathrm{~mm}$; Pixel Spacing $\mathrm{Y}=1.0547000169754028 \mathrm{~mm}$, slice thickness $=1.2000000476837158 \mathrm{~mm}$; echo time $(\mathrm{TE}) 2.859 \mathrm{~ms}$, inversion time (TI) $0.0 \mathrm{~ms}$, repetition time (TR) $6.6764 \mathrm{~ms}$ and weighting T1. We selected subjects as specified in Table.

\section{Subjects}

We selected 93 subjects from ADNI2 cohort. The purpose of ADNI2 is to examine how brain imaging and other biomarkers can be used to measure the progression of MCI and early AD. The ADNI selects and categorizes participants in specific group based on certain inclusion criteria. The criteria are well defined $\mathrm{in}^{2}$. We selected the subjects according to availability of both MRI and fMRI data. Thus, the subjects with following demographic status as shown in Table 1 with following average age, clinical dementia rating (CDR) and mini-mental state estimation (MMSE) out of all available data in ADNI2 cohort were selected in our study.

1. 31 HC subjects: 14 males, 17 females; age $\pm \mathrm{SD}=73.9 \pm 5.4$ years with the mini-mental state estimation (MMSE) score of $28.9 \pm 1.65$ and the range was $24-30$.

2. 31 MCI subjects: 17 males, 14 females; age $\pm \mathrm{SD}=74.5 \pm 5.0$ with the MMSE score of $27.5 \pm 2.02$, and range was $22-30$.

3. 31 AD subjects: 13 males, 18 females; age $\pm \mathrm{SD}=72.7 \pm 7.0$ with $\mathrm{MMSE}=20.87 \pm 3.6$, and the range was 14-26.

\section{Data Preprocessing}

We used data processing subordinate for the resting state fMRI via DPARSF ${ }^{3}$ (Chao-Gan and Yu-Feng, 2010) and the statistical parametric mapping platform via $\mathrm{SPM}^{4}$ aimed at the preprocessing of rs-fMRI data. All the images initially obtained from scanner were in the format of digital imaging and communications in medicine (DICOM). We converted these images to neuroimaging informatics technology initiative (NIfTI) file format. Signal standardization and participant's adaptation to the noise while scanning each participant are carried out by discarding the first 10 time points for each participant. Next, we preformed preprocessing operation through following steps:

For slice-timing correction last slice was referred reference slice. Friston 24-parameter model with 6 parameters of head motion, 6 parameters of head motion from the previous time point, and 12 corresponding squared items were employed for

${ }^{2}$ https://www.nia.nih.gov/alzheimers/clinical-trials/alzheimers-diseaseneuroimaging-initiative-2-adni2

${ }^{3}$ http://www.restfmri.net

${ }^{4}$ http://www.fil.ion.ucl.ac.uk/spm

TABLE 1 | Summary of subject's demographic status.

\begin{tabular}{lccc}
\hline Number of & HC $(\boldsymbol{n}=\mathbf{3 1})$ & MCI $(\boldsymbol{n}=\mathbf{3 1 )}$ & AD $(\boldsymbol{n}=\mathbf{3 1})$ \\
\cline { 2 - 4 } subjects & Mean (SD) & Mean (SD) & Mean (SD) \\
\hline Age (years) & $73.9 \pm 5.4$ & $74.5 \pm 5.0$ & $\mathrm{SD}=72.7 \pm 7.0$ \\
Global CDR & $0.04 \pm 0.13$ & $0.5 \pm 0.18$ & $0.95 \pm 0.30$ \\
MMSE & $28.9 \pm 1.65$ & $27.5 \pm 2.02$ & $20.87 \pm 3.6$
\end{tabular}


realignment for head movement compensation. Similarly, after the realignment, individual structural images (T1-weighted MPRAGE) were registered to the mean functional image. For the standardization of the rs-fMRI toward the original place was accomplished with the help of diffeomorphic anatomical registration through exponentiated lie algebra (DARTEL) as in Ashburner (2007) (resampling voxel size $=3 \mathrm{~mm} \times 3 \mathrm{~mm} \times 3 \mathrm{~mm})$. A $6 \mathrm{~mm}$ full width at half-maximum (FWHM) Gaussian kernel spatial smoothing was employed for the smoothing. Next, we performed linear trend exclusion and also the temporal band pass filtering which ranges at $(0.01 \mathrm{~Hz}<\mathrm{f}<0.08 \mathrm{~Hz})$ on the time series of each voxel. Finally, cerebrospinal as well as white matter signals along with six head-motion parameters were regressed out to reduce the effects of nuisance signals.

\section{Proposed Framework}

This proposed method consists of the following four major functional steps as shown in Figure 1:

1. Construct a brain network using graph theory.

2. Convert graph to feature vector using node2vec graph embedding.

3. Reduce the features.

4. Perform the classification using regularized extreme learning machine (RELM) and linear support vector machine (LSVM).

\section{Construction of Brain Networks}

For the construction of network from fMR images, we first preprocessed the raw fMR data as described in data preprocessing section. Next, we used the automated anatomical labeling (AAL) atlas to identify the brain regions of interest (ROI). The whole image was divided in 116 regions with each hemisphere. Next, we calculate the average time series of each ROI for each subject by averaging their time series across the voxels within each ROI. For each subject, a matrix of 130 rows and 116 columns was obtained. In the matrix, every row denotes the time series conforming to a give ROI, while information of total regions at a definite time point are stored at each column. The mean time series of each brain region were obtained for each individual by averaging the time series within the region. For $L_{i}=\left(l_{i}(1), l_{i}(2), \ldots, l_{i}(n)\right)$ and $L_{j}=\left(l_{j}(1), l_{j}(2), \ldots, l_{j}(n)\right)$ are two $n$ length time series of brain region $i$ and $j$, the Pearson's correlation (PC) between them can be calculated as

$$
P C_{i j}=\frac{\operatorname{cov}\left(L_{i}, L_{j}\right)}{\sigma_{L_{i}} \sigma_{L_{j}}}
$$

Where $\operatorname{cov}\left(L_{i}, L_{j}\right)$ is covariance of variables $L_{i}$ and $L_{j}$. Similarly, $\sigma_{L_{i}}$ and $\sigma_{L_{j}}$ are standard deviation of variables $L_{i}$ and $L_{j}$. This operation results into $116 \times 116$ correlation matrix which defines the relation amongst different regions of brain and matches to the functional connectivity network.

\section{Graph-Embedding}

Graphs are complex data structures, consisting a finite set of vertices and set of edges which connect a pair of nodes. One of the possible solutions to manipulate prevalent pattern recognition algorithms on graphs is embedding the graph into vector space. Indeed, graph embedding is a bridge between statistical pattern recognition and graph mining. We employ the node2vec (Grover and Leskovec, 2016) algorithm as graph embedding tool in this study. The node2vec algorithm aims to learn a vectorial representation of nodes in a graph by optimizing a neighborhood preserving objective. It extends the previous node embedding algorithm Deepwalk (Canu et al., 2010) and it is inspired from the state of art word embedding algorithm word2vec (Delbeuck et al., 2003).

In word2vec, given a set of sentences also known as corpus, the model learns word embedding by analyzing the context of each word in the body. The word2vec uses the neural network with one hidden layer to transform words into embedding vectors. This neural network is known as Skip-gram. This network is trained to

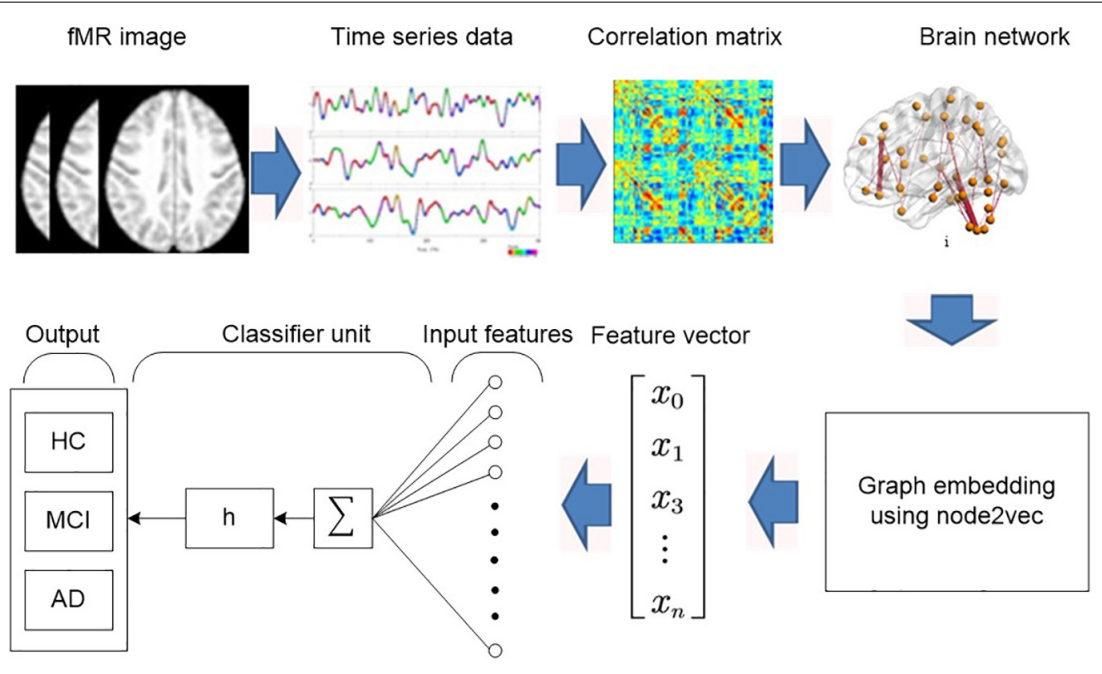

FIGURE 1 | Block diagram of the proposed diagnosis system. 
predict the neighboring word in the sentence. It accepts the word at the input and is optimized such that it predicts the neighboring words in a sentence with high probability.

node2vec applies the same embedding approach to train and predict the neighborhood of a node in graph. However, word is replaced by the node and the bag of nodes is used instead of corpus. The sampling is used to generate this bag of nodes from a graph. Generally, the graph embedding consists of three steps:

\section{Sampling}

A graph is sampled with random walks. This random walk results in bag of nodes of neighborhood from sampling. The bag of nodes acts as a collection of contexts for each node in the network. The innovation of node2vec with respect to Deepwalk is the use of flexible biased random walks on the network. In Deepwalk, random walk is obtained by a uniform random sampling over the linked nodes, while node2vec combine two different strategies for the network exploration: depth-first search (DFS) and breadthfirst-search (BFS). For current random walk position at node $v$ and traversed position at previous step at node $t$ and neighboring nodes $x_{1}, x_{2}$ and $x_{3}$, the sampling of next node $x$ is determined by evaluating the unnormalized transition probabilities $\pi_{v x}$ on edge $(t, v)$ with the static edge weight $w_{v x}$ as shown in Figure 2. This unnormalized transition probability is estimated based on search bias $\alpha$ defined by two parameters $p$ and $q$ such that $\pi_{v x}=$ $\alpha_{p q}(t, x) \cdot w_{v x}$ where.

$$
\alpha_{p q}(t, x)= \begin{cases}\frac{1}{p}, & \text { if } d_{t x}=0 \\ 1, & \text { if } d_{t x}=1 \\ \frac{1}{q}, & \text { if } d_{t x}=2\end{cases}
$$

Here $d_{t x}$ denotes the shortest path distance between nodes $t$ and $x$.

The parameter $p$ determines the likelihood of sampling the node $t$ again during random walk. When the value of $p$ is high

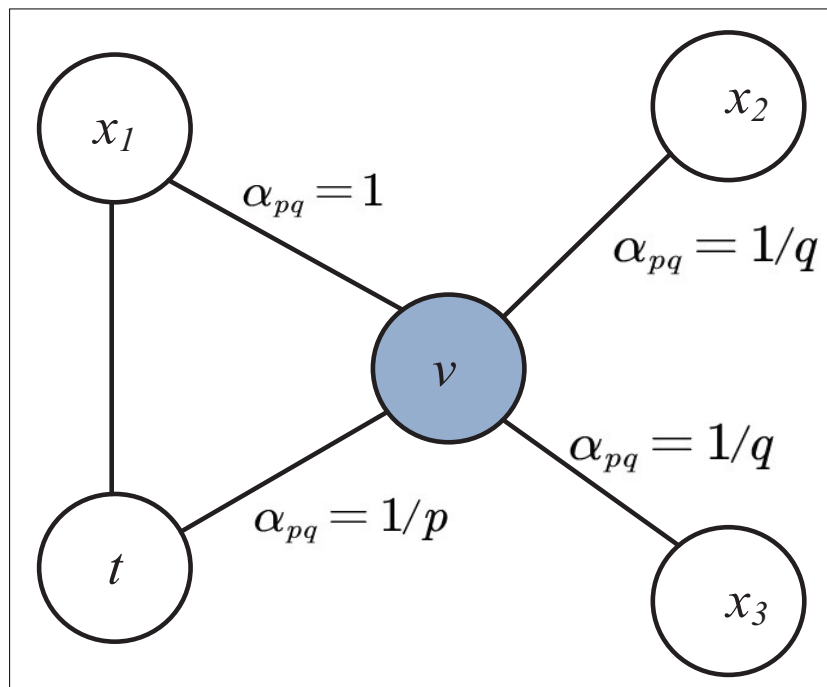

FIGURE 2 | Illustration of node selection in node2vec algorithm. revisit of the node possibility is low. Similarly the parameter $q$ allows to different between local and global nodes. If $q>1$, the random walk has the likelihood of sampling the nodes around the node $v$ is high.

\section{Training Skip-Gram}

The bag of nodes generated from the random walk is fed into the skip-gram network. Each node is represented by a one-hot vector and maximizes the probability for predicting neighbor nodes. The one-hot vector has size same as the size of the set of unique words used in the text corpus. For each node only one dimension is equal to one and remaining are zeros. The position of dimension having one in vector defines the individual node.

\section{Computing Embedding}

The output of the hidden layer of the network is taken as the embedding of the graph.

\section{Feature Reduction Techniques Support Vector Machine-Recursive Feature Elimination (SVM-RFE)}

Support vector machine-recursive feature elimination is a multivariate feature reduction algorithm is based on wrapper model. This method is recursive and in each of iteration of the RFE, LSVM model is trained. This method starts by constructing a model on the complete set of features and computing the importance score for each feature. The least important features are removed and the model is rebuilt and the importance scores are again computed. This recursive procedure is continued until all the features are eliminated. Then, the features are ranked according to the order of elimination. A detailed description of SVM-RFE procedure presented in a previous paper (Guyon et al., 2002). In this work, after applying SVM-RFE, the most significant training features that make the most of cross-validated accurateness are kept to train the classifiers.

\section{Least Absolute Shrinkage and Selection Operator (LASSO)}

Least absolute shrinkage and selection operator (Tibshirani, 1996) is a powerful method which is used to remove insignificant features. Two major tasks of this method are regularization and feature selection. This method minimizes residual sum of squares of the model using ordinary least square regression (OLS) by placing a constraint on the sum of the absolute values of the model parameters. LASSO computes model coefficients $\beta$ by minimizing the following function:

$$
\begin{aligned}
& \operatorname{RSS}_{\text {LASSO }}\left(\beta_{i}, \beta_{0}\right) \\
& =\underset{\beta}{\arg \min }\left[\sum_{i=1}^{n}\left(y_{i}-\left(\beta_{i} x_{i}+\beta_{0}\right)\right)^{2}+\alpha \sum_{j=1}^{k}\left|\beta_{j}\right|\right]
\end{aligned}
$$

Where, $x_{i}$ is the graph embedded feature input data, a vector of $k$ values at observation $j$, and $n$ is the number of observations. $y_{i}$ is the response at observation $i . \alpha$ is a non-negative user defined regularization parameter. This parameter controls the strength of penalty. When $\alpha$ is sufficiently large then coefficients are forced 
to be zero which leads to produce few relevant features. If $\alpha$ approaches 0 the model becomes OLS with more relevant features (Hanyu et al., 2010).

\section{Features Selection With Adaptive Structure Learning (FSASL)}

Features selection with adaptive structure learning is an unsupervised method which performs data manifold learning and feature selection. This method first utilizes the adaptive structure of the data to construct the global learning and the local learning. Next, the significant features are selected by integrating both of them with $L_{2,1}$-norm regularizer. This method utilizes the sparse reconstruction coefficients to extract the global structure of data for global learning. In sparse representation, each data sample $x_{i}$ can be approximated as a linear combination of all the other samples, and the optimal sparse combination weight matrix.

For local learning, this method directly learns a Euclidean distance induced probabilistic neighborhood matrix (Du and Shen, 2015).

$$
\begin{aligned}
& \min _{W, S, P}\left(\left\|W^{T} X-W^{T} X S\right\|^{2}+\alpha\|S\|_{1}\right) \\
& \quad+\beta \sum_{i, j}^{n}\left(\left\|W^{T} x_{i}-W^{T} x_{j}\right\|^{2} P_{i j}+\mu P_{i j}^{2}\right)+\gamma\|W\|_{21} \\
& \quad \text { s.t. } S_{i i}=0, P 1_{n}=1_{n}, P \geq 0, W^{T} X X^{T} X=I
\end{aligned}
$$

Where, $\alpha$ is used to balancing the sparsity and the reconstruction error, $\beta$ and $\gamma$ are regularization parameters for global and local structure learning in first and second group and the sparsity of feature selection matrix in the third group. Similarly, $S$ is used to guide the search of relevant global feature and $P$ defines the local neighborhood of data sample $x_{i}$.

\section{Local Learning and Clustering Based Feature Selection (LLCFS)}

LLCFS is clustering based feature selection method. This method learns the adaptive data structure with selected features by constructing the k-nearest neighbor graph in the weighted feature space (Zeng and Cheung, 2011). The joint clustering and feature weight learning is performed by solving the following problem.

$$
\begin{aligned}
& \min _{Y,\left\{W^{i}, b^{i}\right\}_{i=1}^{n}, z} \sum_{i=1}^{n} \sum_{c^{\prime}=1}^{c}\left[\sum_{x_{j} \in N_{x_{i}}} \beta\left(Y_{i c^{\prime}}-x_{j}^{T} W_{c^{\prime}}^{i}-b_{c}^{i}\right)^{2}\right. \\
& \left.+\left(W_{c^{\prime}}^{i}\right)^{T} \operatorname{diag}\left(z^{-1}\right) W_{c^{\prime}}^{i}\right] \\
& \text { s.t. } 1_{d}^{T} z=1, z \geq 0
\end{aligned}
$$

Where $z$ the feature weight vector and $N_{x_{i}}$ is the k-nearest neighbor of $x_{i}$ based on $z$ weighted features.

\section{Pairwise Correlation Based Feature Selection (CFS)}

CFS selects features based on the ranks attributes according to an empirical evaluation function based on correlations (Hall, 2000).
Subsets made of attribute vectors are evaluated by evaluation function, which are associated with the labels of class, however autonomous among each another. CFS accepts that unrelated structures express a low correspondence with the class and hence they are ought to be overlooked by the procedure. Alternatively, additional features must be studied, as they are typically hugely correlated with one or additional amount of other features.

\section{Classification}

Two of the prevalent machine-learning classification algorithms namely, LSVM, and RELM are studied in this article. The results acquired through the experiments of these classifiers show that RELM classifier performs better than others respective of the computation time required and accuracy value. Each of the methods is described in brief in the subsections below.

\section{Support Vector Machine Classifier}

Linear support vector machine (Cortes and Vapnik, 1995) is principally a supervised binary classifier that classifies separable and non-separable data. This type of classification is usually used in the field of neuroimaging and is deliberated as one of the finest machine-learning method in the domain of the neuroscience for past decades. It discovers the best hyperplane to split both classes which has optimum boundary from support vectors for the duration of the training. The classifier decides on the basis of the estimated hyperplane to test the new data points. For the patterns that are linearly separable, LSVM can be used. Alternatively, LSVM is not capable of guaranteeing improved performance in the complex circumstances with the patterns that are not separable. In such circumstances, Kernel trick is used to extend the LSVM. The input arrays of linear SVM are plotted to the space dimensions using the kernels. Both the linear as well as nonlinear radial basis function (RBF) kernels are extensively trained using SVM kernels.

\section{Extreme Learning Machine}

ELM (Extreme Learning Machine) is single layer feedforward neural networks (Huang et al., 2006; Zhang et al., 2015). This neural network is implemented using Moore-Penrose generalized inverse to set its weights (Peng et al., 2013; Cao et al., 2016). Thus, this learning algorithm doesn't require iterative gradientbased backpropagation to tune the artificial hidden nodes. Thus this method is considered as effective solution with extremely reduced complexity (Cambria and Huang, 2013; Qureshi et al., 2016). ELM with $L$ number of hidden nodes and $\mathrm{g}(\mathrm{x})$ as activation function is expressed as

$$
Y_{L}(x)=\sum_{i=1}^{L} \beta_{i} h_{i}(x)=h(x) \beta_{i}
$$

Where $x$ is an input vector. $h_{i}(x)$ is the input to output node from hidden node output. $\beta=\left[\beta_{1}, \ldots \ldots, \beta_{2}\right]^{T}$ is the weight matrix of $i^{\text {th }}$ node. The input weight $w_{i}$ and the hidden layer biases $b_{i}$ are generated randomly before the training samples are acquired. Thus iterative back-propagation to tune these parameters is not 
needed. Given $\mathrm{N}$ training samples $\left\{\left(x_{j}, t_{j}\right)\right\}_{j=1}^{N}$. The objective function of ELM is expressed as,

$$
\left\|H\left(w_{1}, \ldots . w_{\tilde{N}}, b_{1}, \ldots ., b_{\tilde{N}}\right) \hat{\beta}-T\right\|=\min _{\beta}\|H \hat{\beta}-T\|
$$

with

$$
\begin{gathered}
H\left(w_{1}, \ldots . w_{\tilde{N}}, b_{1}, \ldots, b_{\tilde{N}}\right) \\
=\left[\begin{array}{ccc}
g\left(w_{1} \cdot x_{1}+b_{1}\right) & \cdots & g\left(w_{L} \cdot x_{1}+b_{L}\right) \\
\vdots & \cdots & \vdots \\
g\left(w_{1} \cdot x_{N}+b_{1}\right) & \cdots & g\left(w_{L} \cdot x_{N}+b_{L}\right)
\end{array}\right] \\
, \beta=\left[\begin{array}{c}
\beta_{1}^{T} \\
\vdots \\
\beta_{L}^{T}
\end{array}\right] T=\left[\begin{array}{c}
t_{1}^{T} \\
\vdots \\
t_{L}^{T}
\end{array}\right]
\end{gathered}
$$

Here $H$ represents the hidden layer output matrix and $T$ represents output label of training data matrix. The output weight matrix $\beta$ is calculated as

$$
\beta=H^{+} T
$$

Here, $\mathrm{H}^{+}$represents the Moore-Penrose generalized inverse of the matrix $H$. Since ELM learning approach requires no backpropagation, this method is best suited for the binary and multiclass classification of big data and neuroimaging features. However the decrease in computation time comes with the expense of increase in the error in the output, which ultimately decreases the accuracy. Thus, a regularization term is added to improve generalization performance and make the solution more robust. The output weight of the regularized ELM can be expressed as

$$
\beta=\left(\frac{I}{C}+H^{T} H\right)^{-1} H^{T} T
$$

\section{Performance Evaluation}

We evaluated the performance using the SVM and RELM classifiers for each specific test including the binary and multiclass test. Confusion matrix is constructed to visualize the performance of the binary classifier in a form of a as shown in Table 2 . Correct numbers of prediction of classifier are placed on the diagonal of the matrix. These components are further divided into true positive (TP), true negative (TN), which represent correctly identified controls. Similarly, the false positive (FP) and false negative (FN) represent the number of wrongly classified subjects.

TABLE 2 | Confusion matrix.

\begin{tabular}{lll}
\hline Accurate Class & \multicolumn{2}{c}{ Predicted Class } \\
\cline { 2 - 3 } & C1 & C2 \\
\hline C1 & TP & FN \\
C2 & FP & TN
\end{tabular}

The proportion of subjects which are correctly classified by the classifier is expressed as the accuracy.

$$
A C C=\frac{T P+T N}{T P+T N+F P+F N}
$$

However, for dataset with unbalanced class distribution accuracy may be a good performance metric. Thus two more performance are used. These metrics are known as sensitivity and specificity are used.

$$
\begin{aligned}
& S E N=\frac{T P}{T P+F N} \\
& S P E=\frac{T N}{T N+F P}
\end{aligned}
$$

The sensitivity (SEN) measures the rate of true positives (TP) while the specificity (SPE) measures rate of true negatives (TN).

\section{RESULTS}

\section{Demographic and Clinical Findings}

We did not find a significant group difference in age in $\mathrm{AD}$ versus $\mathrm{HC}, \mathrm{AD}$ versus $\mathrm{MCI}$ and MCI versus $\mathrm{HC}$. However significant group difference was found in MMSE $(P<0.01)$ and CDR $(P<0.01)$ in all group combinations. The gender proportion on both $\mathrm{AD}$ and $\mathrm{HC}$ is male dominant. $\mathrm{AD}$ has $54.83 \%$ and $\mathrm{HC}$ has $45.16 \%$ male dominance. Table 1 shows the detailed descriptions and analysis of these variables.

\section{Classification Results}

We have observed the performance of our proposed algorithm and compared it with that of the RELM classifier and LSVM classifier for respective test comprising the binary classification. The performance shown by the binary classifier is envisaged as a confusion matrix as presented in Table 1. Elements on the diagonal elements of the matrix specify the accurate estimations by the classifier. These elements are further divided as true positive (TP) and true negative (TN), which signifies appropriately recognized controls. Correspondingly, all the erroneously classified matters can be symbolized by false positive (FP) and false negative (FN). We evaluated the feature selection and classification algorithms on data set using a 10-flold cross validation (CV). First, we divided the subjects into 10 equally sized subsets: each of these subsets (folds), containing $10 \%$ of the subjects as test set and remaining $90 \%$ for training set. Then feature ranking was performed on the training sets. We used different algorithms to rank the features. Linear SVM and RELM classifiers were trained using these top-ranked features. For each training and test we performed separate feature selection to avoid the feature selection bias during 10-fold cross validation. We calculated cross validated average classification accuracy and standard deviation for specific feature using $k$-top most ranked features, where $k$ ranges from 1 to 50 . We repeatedly tested for 5 iterations and plotted the mean accuracy and standard deviation as shown in Figure 3 for LASSO feature selection and RELM classifier. 


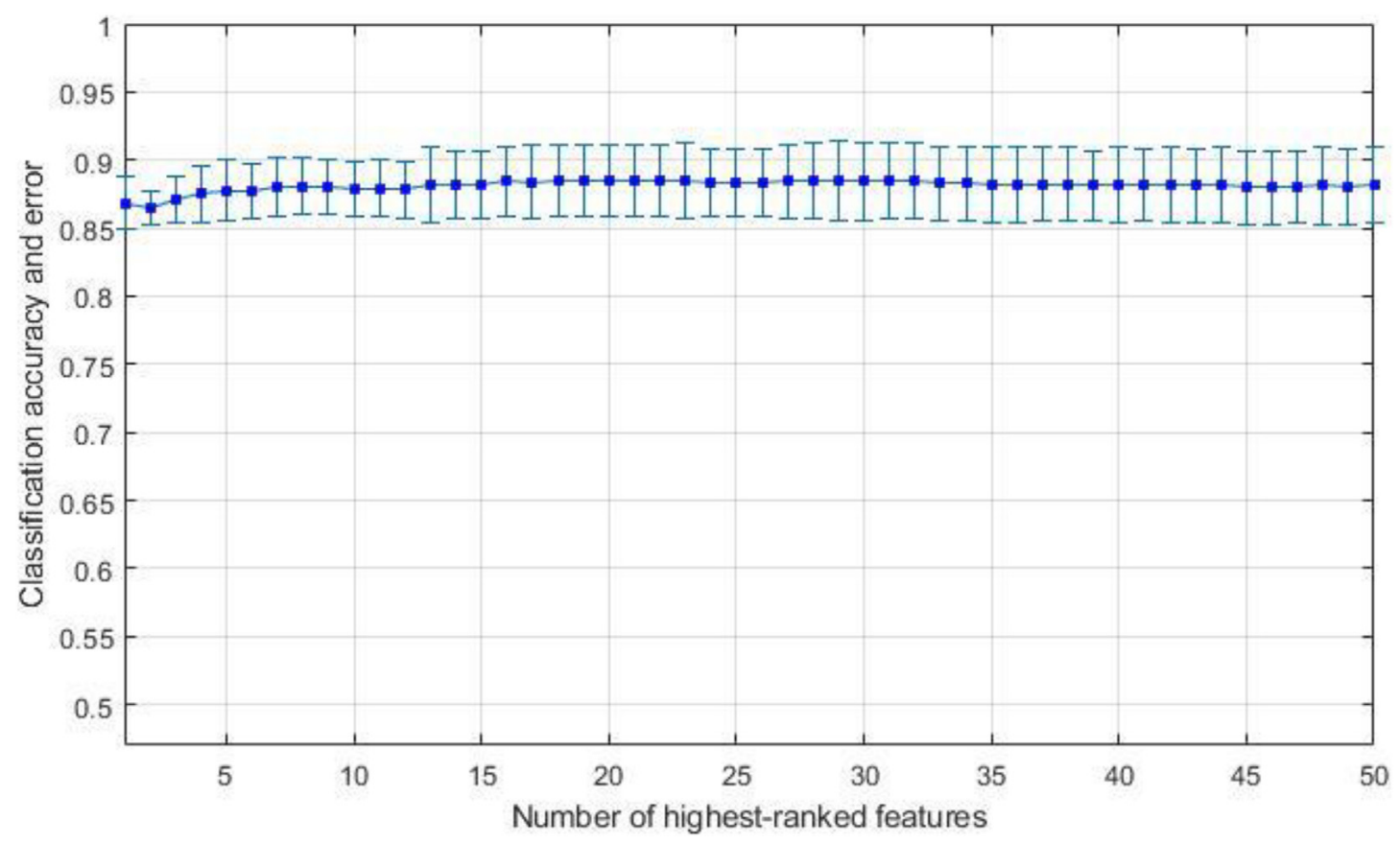

FIGURE 3 | Average accuracy and standard deviation for AD against HC using RELM classification method on reduced datasets using LASSO feature selection.

TABLE 3 | 10-fold cross-validation binary mean classification performance for AD against $\mathrm{HC}$ using RELM classifier using different feature selection methods.

\begin{tabular}{|c|c|c|c|c|c|}
\hline $\begin{array}{l}\text { Feature } \\
\text { selection } \\
\text { method }\end{array}$ & $\begin{array}{l}\text { Performance } \\
\text { metrics }\end{array}$ & Mean (\%) & $\begin{array}{l}\text { Standard } \\
\text { deviation }\end{array}$ & $\operatorname{Max}(\%)$ & Min (\%) \\
\hline \multirow[t]{4}{*}{ LASSO } & Accuracy & 87.723 & 0.468 & 88.663 & 85.82 \\
\hline & Sensitivity & 90.93 & 0.341 & 91.525 & 89.50 \\
\hline & Specificity & 84.52 & 0.792 & 85.891 & 82.14 \\
\hline & F-measure & \multicolumn{4}{|c|}{0.883} \\
\hline \multirow[t]{4}{*}{ FSASL } & Accuracy & 76.181 & 1.069 & 78.551 & 73.45 \\
\hline & Sensitivity & 76.233 & 1.255 & 78.839 & 72.58 \\
\hline & Specificity & 75.664 & 1.264 & 77.868 & 72.20 \\
\hline & F-measure & \multicolumn{4}{|c|}{0.785} \\
\hline \multirow[t]{4}{*}{ LLCFS } & Accuracy & 75.737 & 1.004 & 78.690 & 71.64 \\
\hline & Sensitivity & 74.205 & 1.069 & 77.031 & 70.11 \\
\hline & Specificity & 77.881 & 1.378 & 81.036 & 73.64 \\
\hline & F-measure & \multicolumn{4}{|c|}{0.817} \\
\hline \multirow[t]{4}{*}{ CFS } & Accuracy & 80.517 & 1.737 & 82.86 & 74.005 \\
\hline & Sensitivity & 80.035 & 1.813 & 82.22 & 73.25 \\
\hline & Specificity & 79.16 & 1.977 & 81.79 & 73.084 \\
\hline & F-measure & \multicolumn{4}{|c|}{0.867} \\
\hline \multirow[t]{4}{*}{ SVM-RFE } & Accuracy & 68.57 & 1.186 & 70.474 & 65.60 \\
\hline & Sensitivity & 75.99 & 1.676 & 78.832 & 71.715 \\
\hline & Specificity & 60.92 & 1.301 & 63.426 & 57.34 \\
\hline & F-measure & \multicolumn{4}{|c|}{0.6743} \\
\hline
\end{tabular}

Finally, we calculated the mean accuracy and standard deviation of highest ranked features for different feature selection and classification methods as depicted in Tables
TABLE 4 | 10-fold cross-validation binary mean classification performance for HC against $\mathrm{MCl}$ using RELM classifier using different feature selection methods.

\begin{tabular}{|c|c|c|c|c|c|}
\hline $\begin{array}{l}\text { Feature } \\
\text { selection } \\
\text { method }\end{array}$ & $\begin{array}{l}\text { Performance } \\
\text { metrics }\end{array}$ & Mean (\%) & $\begin{array}{l}\text { Standard } \\
\text { deviation }\end{array}$ & $\operatorname{Max}(\%)$ & Min (\%) \\
\hline \multirow[t]{4}{*}{ LASSO } & Accuracy & 96.11 & 0.859 & 96.88 & 91.33 \\
\hline & Sensitivity & 95.03 & 1.080 & 95.93 & 89.84 \\
\hline & Specificity & 97.18 & 0.798 & 97.84 & 92.93 \\
\hline & F-measure & \multicolumn{4}{|c|}{0.973} \\
\hline \multirow[t]{4}{*}{ FSASL } & Accuracy & 85.85 & 0.9129 & 87.503 & 80.88 \\
\hline & Sensitivity & 79.27 & 0.986 & 81.484 & 76.01 \\
\hline & Specificity & 92.03 & 1.4433 & 93.308 & 85.40 \\
\hline & F-measure & \multicolumn{4}{|c|}{0.937} \\
\hline \multirow[t]{4}{*}{ LLCFS } & Accuracy & 82.29 & 0.624 & 83.39 & 80.77 \\
\hline & Sensitivity & 77.54 & 0.73 & 78.566 & 75.408 \\
\hline & Specificity & 86.81 & 1.081 & 88.41 & 83.74 \\
\hline & F-measure & \multicolumn{4}{|c|}{0.85} \\
\hline \multirow[t]{4}{*}{ CFS } & Accuracy & 80.67 & 1.68 & 90.427 & 74.48 \\
\hline & Sensitivity & 88.43 & 2.328 & 74.968 & 80.49 \\
\hline & Specificity & 72.38 & 1.3677 & 74.486 & 68.58 \\
\hline & F-measure & \multicolumn{4}{|c|}{0.795} \\
\hline \multirow[t]{4}{*}{ SVM-RFE } & Accuracy & 80.20 & 0.920 & 82.001 & 77.89 \\
\hline & Sensitivity & 84.00 & 1.207 & 85.99 & 79.86 \\
\hline & Specificity & 75.91 & 1.251 & 77.806 & 73.29 \\
\hline & F-measure & \multicolumn{4}{|c|}{0.815} \\
\hline
\end{tabular}

3-8 and the bold values in each table indicate the maximum value of accuracy, sensitivity and specificity. Maximum and minimum value of accuracy, sensitivity 
TABLE 5 | 10-fold cross-validation binary mean classification performance for $\mathrm{MCl}$ against $A D$ using RELM classifier using different feature selection methods.

\begin{tabular}{|c|c|c|c|c|c|}
\hline $\begin{array}{l}\text { Feature } \\
\text { selection } \\
\text { method }\end{array}$ & $\begin{array}{l}\text { Performance } \\
\text { metrics }\end{array}$ & Mean (\%) & $\begin{array}{l}\text { Standard } \\
\text { deviation }\end{array}$ & $\operatorname{Max}(\%)$ & Min (\%) \\
\hline \multirow[t]{4}{*}{ LASSO } & Accuracy & 93.86 & 0.766 & 94.90 & 89.128 \\
\hline & Sensitivity & 91.93 & 0.757 & 93.67 & 89.836 \\
\hline & Specificity & 95.92 & 1.204 & 96.61 & 88.580 \\
\hline & F-measure & \multicolumn{4}{|c|}{0.968} \\
\hline \multirow[t]{4}{*}{ FSASL } & Accuracy & 85.358 & 1.030 & 86.76 & 80.29 \\
\hline & Sensitivity & 85.088 & 0.951 & 86.61 & 80.47 \\
\hline & Specificity & 85.201 & 1.4227 & 86.829 & 79.27 \\
\hline & F-measure & \multicolumn{4}{|c|}{0.879} \\
\hline \multirow[t]{4}{*}{ LLCFS } & Accuracy & 90.32 & 1.06316 & 91.88 & 86.50 \\
\hline & Sensitivity & 93.33 & 2.0782 & 95.63 & 87.43 \\
\hline & Specificity & 87.49 & 0.9471 & 89.56 & 85.75 \\
\hline & F-measure & \multicolumn{4}{|c|}{0.895} \\
\hline \multirow[t]{4}{*}{ CFS } & Accuracy & 79.13 & 1.2768 & 81.595 & 75.10 \\
\hline & Sensitivity & 83.59 & 1.2281 & 85.60 & 78.94 \\
\hline & Specificity & 74.83 & 1.7084 & 78.927 & 70.54 \\
\hline & F-measure & \multicolumn{4}{|c|}{0.795} \\
\hline \multirow[t]{4}{*}{ SVMRFE } & Accuracy & 77.5974 & 0.8177 & 78.93 & 74.98 \\
\hline & Sensitivity & 76.802 & 1.1299 & 79.225 & 73.26 \\
\hline & Specificity & 78.182 & 0.8359 & 79.289 & 75.44 \\
\hline & F-measure & \multicolumn{4}{|c|}{0.8169} \\
\hline
\end{tabular}

TABLE 6 | 10-fold cross-validation binary mean classification performance for AD against HC using LSVM classifier using different feature selection methods.

\begin{tabular}{|c|c|c|c|c|c|}
\hline $\begin{array}{l}\text { Feature } \\
\text { selection } \\
\text { method }\end{array}$ & $\begin{array}{l}\text { Performance } \\
\text { metrics }\end{array}$ & Mean (\%) & $\begin{array}{l}\text { Standard } \\
\text { deviation }\end{array}$ & Max (\%) & Min (\%) \\
\hline \multirow[t]{4}{*}{ LASSO } & Accuracy & 90.63 & 0.515 & 91.51 & 88.52 \\
\hline & Sensitivity & 87.044 & 0.585 & 88.03 & 85.44 \\
\hline & Specificity & 94.315 & 0.671 & 95.35 & 90.95 \\
\hline & F-measure & \multicolumn{4}{|c|}{0.958} \\
\hline \multirow[t]{4}{*}{ FSASL } & Accuracy & 82.895 & 1.4020 & 85.60 & 80.19 \\
\hline & Sensitivity & 78.206 & 1.5118 & 81.99 & 75.21 \\
\hline & Specificity & 87.712 & 1.7666 & 90.02 & 82.85 \\
\hline & F-measure & \multicolumn{4}{|c|}{0.8360} \\
\hline \multirow[t]{4}{*}{ LLCFS } & Accuracy & 81.19 & 1.438 & 83.37 & 77.68 \\
\hline & Sensitivity & 85.15 & 2.087 & 88.068 & 80.49 \\
\hline & Specificity & 76.39 & 1.25 & 78.983 & 73.65 \\
\hline & F-measure & \multicolumn{4}{|c|}{0.8095} \\
\hline \multirow[t]{4}{*}{ CFS } & Accuracy & 88.37 & 1.78 & 91.18 & 83.25 \\
\hline & Sensitivity & 87.95 & 1.72 & 91.98 & 84.52 \\
\hline & Specificity & 88.79 & 2.17 & 90.71 & 81.28 \\
\hline & F-measure & \multicolumn{4}{|c|}{0.903} \\
\hline \multirow[t]{4}{*}{ SVMRFE } & Accuracy & 65.99 & 1.48 & 68.51 & 62.05 \\
\hline & Sensitivity & 65.03 & 1.41 & 67.73 & 61.46 \\
\hline & Specificity & 67.40 & 2.327 & 70.53 & 61.27 \\
\hline & F-measure & \multicolumn{4}{|c|}{0.671} \\
\hline
\end{tabular}

and specificity are calculated amongst corresponding values estimated for highest ranked features as shown in Figure 3.
TABLE 7 | 10-fold cross-validation binary mean classification performance for $\mathrm{HC}$ against MCl using LSVM classifier using different feature selection methods.

\begin{tabular}{|c|c|c|c|c|c|}
\hline $\begin{array}{l}\text { Feature } \\
\text { selection } \\
\text { method }\end{array}$ & $\begin{array}{l}\text { Performance } \\
\text { metrics }\end{array}$ & Mean (\%) & $\begin{array}{l}\text { Standard } \\
\text { deviation }\end{array}$ & $\operatorname{Max}(\%)$ & $\operatorname{Min}(\%)$ \\
\hline \multirow[t]{4}{*}{ LASSO } & Accuracy & 98.91 & 0.456 & 99.25 & 95.82 \\
\hline & Sensitivity & 99.68 & 0.56 & 100.0 & 95.48 \\
\hline & Specificity & 98.11 & 0.46 & 98.51 & 96.00 \\
\hline & F-measure & \multicolumn{4}{|c|}{0.9856} \\
\hline \multirow[t]{4}{*}{ FSASL } & Accuracy & 81.28 & 1.010 & 83.01 & 77.64 \\
\hline & Sensitivity & 84.61 & 1.389 & 86.62 & 79.81 \\
\hline & Specificity & 77.92 & 1.121 & 79.81 & 73.038 \\
\hline & F-measure & \multicolumn{4}{|c|}{0.833} \\
\hline \multirow[t]{4}{*}{ LLCFS } & Accuracy & 76.27 & 0.631 & 78.31 & 74.70 \\
\hline & Sensitivity & 71.08 & 1.388 & 75.37 & 68.23 \\
\hline & Specificity & 81.40 & 1.005 & 82.69 & 76.80 \\
\hline & F-measure & \multicolumn{4}{|c|}{0.800} \\
\hline \multirow[t]{4}{*}{ CFS } & Accuracy & 86.16 & 2.25 & 88.96 & 80.47 \\
\hline & Sensitivity & 92.28 & 2.33 & 95.11 & 86.14 \\
\hline & Specificity & 79.72 & 2.375 & 82.75 & 73.88 \\
\hline & F-measure & \multicolumn{4}{|c|}{0.8517} \\
\hline \multirow[t]{4}{*}{ SVMRFE } & Accuracy & 71.92 & 0.832 & 74.43 & 69.93 \\
\hline & Sensitivity & 66.90 & 1.493 & 71.14 & 63.53 \\
\hline & Specificity & 76.68 & 1.187 & 79.61 & 72.88 \\
\hline & F-measure & \multicolumn{4}{|c|}{0.7762} \\
\hline
\end{tabular}

TABLE 8 | 10-fold cross-validation binary mean classification performance for $\mathrm{MCl}$ against $A D$ using LSVM classifier using different feature selection methods.

\begin{tabular}{|c|c|c|c|c|c|}
\hline $\begin{array}{l}\text { Feature } \\
\text { selection } \\
\text { method }\end{array}$ & $\begin{array}{l}\text { Performance } \\
\text { metrics }\end{array}$ & Mean (\%) & $\begin{array}{l}\text { Standard } \\
\text { deviation }\end{array}$ & Max (\%) & Min (\%) \\
\hline \multirow[t]{4}{*}{ LASSO } & Accuracy & 97.80 & 0.9862 & 98.32 & 91.99 \\
\hline & Sensitivity & 97.62 & 1.0065 & 97.92 & 91.73 \\
\hline & Specificity & 97.74 & 1.0720 & 98.5 & 92.07 \\
\hline & F-measure & \multicolumn{4}{|c|}{0.98} \\
\hline \multirow[t]{4}{*}{ FSASL } & Accuracy & 83.71 & 0.90 & 85.00 & 78.15 \\
\hline & Sensitivity & 90.63 & 1.57 & 92.23 & 84.12 \\
\hline & Specificity & 77.10 & 1.098 & 79.74 & 72.55 \\
\hline & F-measure & \multicolumn{4}{|c|}{0.838} \\
\hline \multirow[t]{4}{*}{ LLCFS } & Accuracy & 90.04 & 1.43 & 92.02 & 86.07 \\
\hline & Sensitivity & 90.23 & 1.43 & 91.94 & 86.74 \\
\hline & Specificity & 90.09 & 1.77 & 92.52 & 84.62 \\
\hline & F-measure & \multicolumn{4}{|c|}{0.903} \\
\hline \multirow[t]{4}{*}{ CFS } & Accuracy & 84.41 & 1.95 & 86.81 & 79.18 \\
\hline & Sensitivity & 90.37 & 2.14 & 93.26 & 83.73 \\
\hline & Specificity & 78.10 & 1.90 & 80.79 & 72.84 \\
\hline & F-measure & \multicolumn{4}{|c|}{0.83} \\
\hline \multirow[t]{4}{*}{ SVMRFE } & Accuracy & 82.87 & 0.903 & 83.96 & 78.832 \\
\hline & Sensitivity & 81.68 & 1.22 & 84.34 & 77.10 \\
\hline & Specificity & 84.11 & 0.94 & 85.21 & 80.72 \\
\hline & F-measure & \multicolumn{4}{|c|}{0.854} \\
\hline
\end{tabular}

Tables 3-5 show the binary classification results using RELM classifier with five different feature selections. Results obtained through the feature selection methods are compared in 

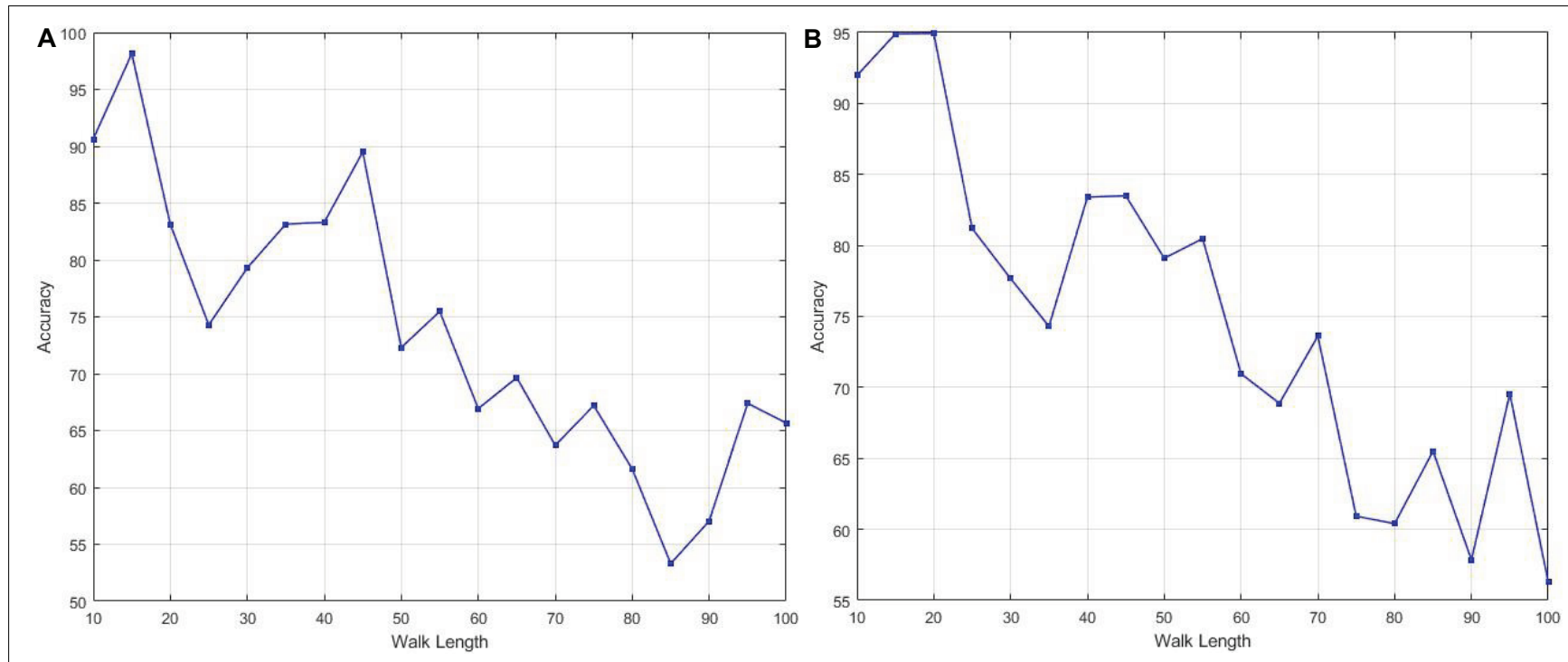

C

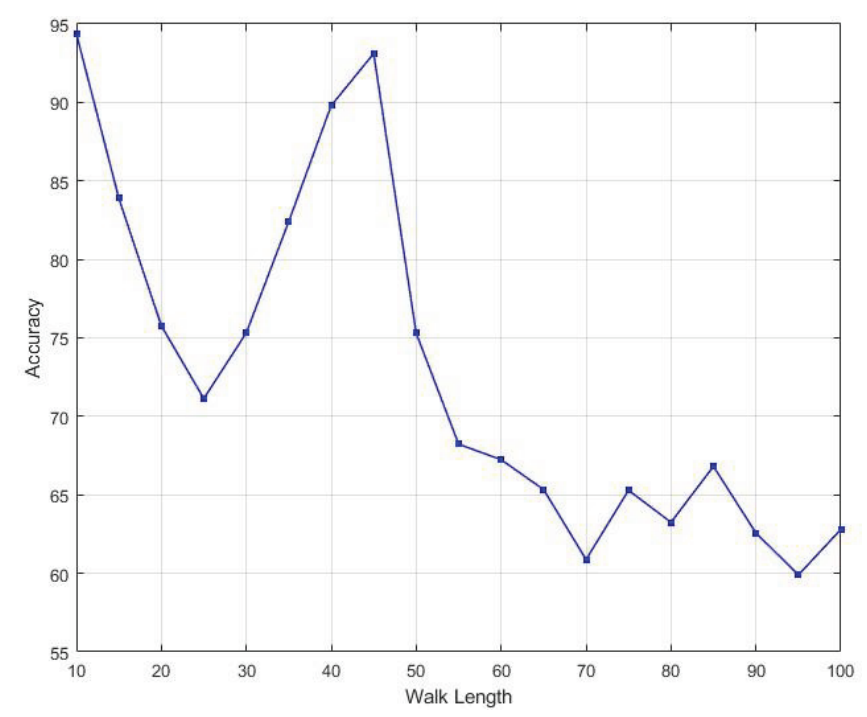

FIGURE 4 | The effect of different parameter values of Walk Length of Node2vec on performance (A) AD against $\mathrm{HC}$, (B) $\mathrm{HC}$ against MCl, (C) AD against MCl.

regards to the performance metrics such as accuracy, sensitivity specificity and f-measure. Table 3 summarizes the AD versus $\mathrm{HC}$ classification. The LASSO feature selection method outperforms all other methods consider with the highest mean accuracy of $87.72 \%$, mean specificity of $90.93 \%$ and mean sensitivity of $84.52 \%$. Additionally, the standard deviation of LASSO is 0.4 which is less than less than 1 . Similarly, the classification results of $\mathrm{AD}$ versus $\mathrm{MCI}$ and $\mathrm{NC}$ versus MCI using RELM are shown in Tables 4, 5. As shown in Table 4, the highest mean accuracy is $96.11( \pm 0.859)$ for HC against MCI classification and $93.86( \pm 0.766)$ for MCI against AD classification. The standard deviation is less than 1 in both mean classifications. Additionally the F-score is high in all three classifications ( 0.883 for $\mathrm{HC}$ against $\mathrm{AD}, 0.973$ for $\mathrm{HC}$ against $\mathrm{MCI}, 0.968$ for $\mathrm{AD}$ against $\mathrm{MCI}$ ) using LASSO feature section method compared to other feature selection methods. The value of standard deviation less than one indicates that the data points of accuracy estimated tend to be close to the mean. Hence from the result it is very evident that the less inflated accuracy can be obtained using LASSO. Similarly, the high F-score indicates precision of classification is high compared to other feature selection methods.

Similarly, the comparison of classification of $\mathrm{HC}$, MCI and AD using LSVM classifier with different feature selection methods are shown in Tables 6-8. Similar to RELM, the highest performance result in terms of mean accuracy, specificity, sensitivity and F-score was obtained by using LASSO for all three classification tests. As shown in Table 6, we obtained the accuracy of $90.63 \%$ specificity of $94.315 \%$ and sensitivity 
of $87.95 \%$ and F-score of 0.958 for $\mathrm{AD}$ against HC. In Table 7 the highest mean accuracy, specificity, sensitivity and F-score are obtained as 98.9, 99.68, 98.11, and $0.9856 \%$ for HC against MCI classification. Similarly, Table 8 shows the classification performance of $\mathrm{AD}$ against MCI. The highest mean accuracy, specificity, sensitivity and F-score are 97.81, 97.62, 97.74, and $0.98 \%$.

From all these results, it is clearly evident that the use of LASSO as feature selection method is ideal choice for the classification using RELM and LSVM classifiers for the graph embedded data.

From Tables 3-5 the highest classification accuracies of RELM classifier using LASSO feature selection for AD against $\mathrm{HC}, \mathrm{HC}$ against MCI and MCI against AD are 87.723\% ( \pm 0.468$), 96.11 \%$ $( \pm 0.859)$, and $93.86 \%( \pm 0.766)$. Similarly, from Tables 6-8 the highest classification accuracies of RELM classifier using LASSO feature selection for $\mathrm{AD}$ against $\mathrm{HC}, \mathrm{HC}$ against $\mathrm{MCI}$ and MCI against $\mathrm{AD}$ are $90.63 \%( \pm 0.515), 98.91 \%( \pm 0.456)$, and $97.80 \%( \pm 0.9862)$.

Now, the comparison of performance between two classifiers shows that the SVM can classify the given dataset more accurately with the highest mean accuracy for all three binary classifications. However, the small standard deviation of the classification HC against MCI and MCI against AD suggest that the classification accuracy values are less inflated in RELM as compared to LSVM.

The number of hidden layer nodes influences the performance of the RELM classifier. In our experiment, we found 1000 number of hidden layer generated the best performance in terms of accuracy. Similarly, for SVM we set the default parameter defined for the MATLAB library. We performed the classification by varying different parameters on node2vec graph embedding. Figure 4 shows the effect of different parameters of node2vec on the performance of RELM classifier. We varied the walk length of node2vec from 10 to 100 . In all experiments, increased value of walk length decreases the performance of classifier. For this experiment, we fixed two other parameters, dimension and number of walks to 32 and 200. Similarly, we set the parameters $p$ and $q$ to correspond localized random walks. With the smaller value of $p$ and larger value of $q$, the random walk is easy to sample to the high-order -order proximity. Thus, we selected $p$ and $q$ randomly and performed graph embedding with $p=0.1$ and $q=1.6$.

\section{DISCUSSION}

Several studies based on rs-fMRI have been carried out for the classification of $\mathrm{AD}$ and MCI from HC subjects. Binary classification in combination of different classifier with different feature measure reported the accuracy ranging from 85 to $95 \%$ for $\mathrm{AD}$ against $\mathrm{HC}$ and 62.90 to $72.58 \%$ to and MCI against $\mathrm{HC}$ as shown in Tables 9, 10. These studies used the same MCI and HC subjects from the ADNI2 cohort. One can clearly notice that the number of subjects directly influences the accuracy. As the number of subjects increase the accuracy is decreased. As reported in previous section the highest accuracy for the classification of $\mathrm{AD}$ from is obtained in proposed work is $90.63 \%$ using the combination of LASSO and LSVM. If we compare the results for MCI against $\mathrm{HC}$, the results obtained in current study outperform all the state of art methods. However, it is not fair to compare performance with other studies directly because each work employ different datasets, preprocessing pipelines, feature measures, and classifiers. Majority of works including (Eavani et al., 2013; Leonardi et al., 2013; Wu et al., 2013; Wee et al., 2014; Suk et al., 2016) have used subjects less than or nearly equal to 30 in each subject class. The main reason behind small number of dataset is the availability of fMRI data in ADNI2 cohort. All of these studies performed classification and made conclusion. Likewise, we also conducted our study using ADNI2 cohort with nearly equal number of subjects with

TABLE 9 | Comparison of performance of binary classification AD against $\mathrm{HC}$ with state of the art methods using rs-fMRI.

\begin{tabular}{|c|c|c|c|c|}
\hline Dataset & Feature measures & Classifier & Accuracy (\%) & Reference \\
\hline AD:77, HC: 173 & Combination of FC matrices, FC dynamics, ALFF & AUC & 85 & de Vos et al., 2018 \\
\hline AD: 12, HC: 12 & Difference between DMN and SN map & LDA & 92 & Zhou et al., 2010 \\
\hline AD: $34, \mathrm{HC}: 45$ & Graph measures & Naïve Bayes & 93.3 & Khazaee et al., 2017 \\
\hline AD: $15, \mathrm{HC}: 16$ & Averaged voxel intensities of core regions in resting state & Multivariate ROC & 95 & Wu et al., 2013 \\
\hline
\end{tabular}
networks: DMN, DAN, VAN

TABLE 10 | Comparison of performance of binary classification $\mathrm{MCl}$ against $\mathrm{HC}$ with state of the art methods using rs-fMRI.

\begin{tabular}{|c|c|c|c|c|}
\hline Dataset & Feature measures & Classifier & Accuracy (\%) & References \\
\hline MCl: 31, HC: 31 & Functional activity co-variations of ROIs & SVM & 62.90 & Eavani et al., 2013 \\
\hline MCl: 31, HC: 31 & Group sparse representation & SVM & 66.13 & Wee et al., 2014 \\
\hline MCl: 31, HC: 31 & SDFN & SVM & 70.97 & Leonardi et al., 2013 \\
\hline MCl: 31, HC: 31 & Deep auto encoder and HMM & SVM & 72.58 & Suk et al., 2016 \\
\hline
\end{tabular}

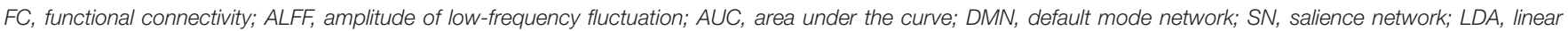

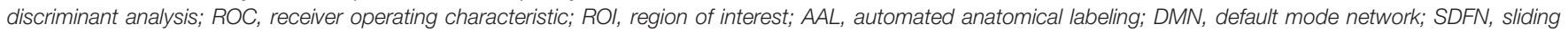
window-based dynamic functional network; HMM, hidden markov model. 
previous studies and the cross validation was also done using these dataset.

Mild cognitive impairment is a transitional stage between the healthy non dementia and dementia stage ${ }^{2}$. This stage is further divided into early MCI (EMCI) and late MCI (LMCI), according to extent of episodic memory impairment. The risk conversion from MCI to AD is higher in LMCI than in EMCI. In this study, we included only EMCI subjects in MCI group. The MCI converted and non-converted to is classified according to CDR and MMSE score. MCI subjects whose CDR undergoes change from 0.5 to 1 and MMSE score goes below 26 in subsequent visits are considered to have fulfilled the criteria to be MCI converted. In our study majority of subjects fulfill to be nonconverted MCI. Only few subjects either have changed CDR score or MMSE score during the visits in the interval of 3,6,12, and 18 months. Additionally, none of the MCI subjects are recorded in the list of $\mathrm{AD}$ subjects.

\section{Limitations}

While this study is focused on the stage diagnosis of $\mathrm{AD}$ progression using fMRI alone using ADNI2 cohort, the major limitation of this study is the limited sample size of ADNI2 (31 $\mathrm{AD}, 31 \mathrm{MCI}$, and $31 \mathrm{HC}$ ). In this context, the entire population is not represented adequately with the dataset we used. Thus, we cannot guarantee the generalization of our results to other groups.

\section{CONCLUSION}

It is widely accepted that the early diagnosis of $\mathrm{AD}$ and MCI plays an import role to take preventive action and to delay the future progression of $\mathrm{AD}$. Thus the accurate classification task of different stages of AD progression is essential. In this study, we demonstrated graph based features from functional magnetic resonance (fMR) images can be used for the classification of $\mathrm{AD}$ and MCI from HC. Additionally, we used multiple feature selection techniques to cope with the smaller number of subjects with larger number of feature representations. The appropriate amount of features is extracted from standard Alzheimer's disease Neuroimaging Initiative cohort that lead to maximal classification accuracies as compared to all other recent researches. Among different feature section methods LASSO together with LSVM on graph based features significantly improved the classification accuracy.

\section{DATA AVAILABILITY STATEMENT}

The raw data supporting the conclusions of this article will be made available by the authors, without undue reservation.

\section{ETHICS STATEMENT}

The studies involving human participants were reviewed and approved by Alzheimer's Disease Neuroimaging Initiative
(ADNI). The patients/participants provided their written informed consent to participate in this study.

\section{AUTHOR CONTRIBUTIONS}

RL has generated the idea and conducted the experiments. G-RK has reviewed idea and final verification of results. All authors contributed to the article and approved the submitted version.

\section{FUNDING}

This work was supported by the National Research Foundation of Korea (NRF) grant funded by the Korea Government (MSIT) (No. NRF-2019R1A4A1029769).

\section{ACKNOWLEDGMENTS}

Data collection and sharing for this project was funded by the Alzheimer's Disease Neuroimaging Initiative (ADNI) (National Institutes of Health Grant U01 AG024904) and DOD ADNI (Department of Defense award number W81XWH12-2-0012). As such, the investigators within the ADNI contributed to the design and implementation of ADNI and/or provided data but did not participate in analysis or writing of this report. A complete listing of ADNI investigators can be found at: http://adni.loni.usc.edu/wpcontent/uploads/how_to_apply/ADNI_Acknowledgement_List. pdf. ADNI is funded by the National Institute on Aging, the National Institute of Biomedical Imaging and Bioengineering, and through generous contributions from the following: AbbVie, Alzheimer's Association; Alzheimer's Drug Discovery Foundation; Araclon Biotech; BioClinica, Inc.; Biogen; BristolMyers Squibb Company; CereSpir, Inc.; Cogstate; Eisai Inc.; Elan Pharmaceuticals, Inc.; Eli Lilly and Company; EuroImmun; F. Hoffmann-La Roche Ltd and its affiliated company Genentech, Inc; Fujirebio; GE Healthcare; IXICO Ltd.; Janssen Alzheimer Immunotherapy Research \& Development, LLC.; Johnson \& Johnson Pharmaceutical Research and Development LLC.; Lumosity; Lundbeck; Merck \& Co., Inc.; Meso Scale Diagnostics, LLC.; NeuroRx Research; Neurotrack Technologies; Novartis Pharmaceuticals Corporation; Pfizer Inc.; Piramal Imaging; Servier; Takeda Pharmaceutical Company; and Transition Therapeutics. The Canadian Institutes of Health Research is providing funds to support ADNI clinical sites in Canada. Private sector contributions are facilitated by the Foundation for the National Institutes of Health (www.fnih.org). The grantee organization is the Northern California Institute for Research and Education, and the study is coordinated by the Alzheimer's Therapeutic Research Institute at the University of Southern California. ADNI data are disseminated by the Laboratory for Neuro Imaging at the University of Southern California. Correspondence should be addressed to GR-K, grkwon@chosun.ac.kr. 


\section{REFERENCES}

Acosta-Cabronero, J., Alley, S., Williams, G. B., Pengas, G., and Nestor, P. J. (2012). Diffusion tensor metrics as biomarkers in Alzheimer's disease. PLoS One 7:e49072. doi: 10.1371/journal.pone.0049072

Alzheimer's association (2016). 2016 Alzheimer's disease facts and figures. Alzheimers Dement. 12, 459-509. doi: 10.1016/j.jalz.2016.03.001

American Psychiatric Association (1994). "Task force on DSM-IV," in Diagnostic and Statistical Manual of Mental Disorders," DSM-IV, 4th Edn, Vol. xxv, (Washington, DC: American Psychiatric Association).

Ashburner, J. (2007). A fast diffeomorphic image registration algorithm. Neuroimage 38, 95-113. doi: 10.1016/j.neuroimage.2007.07.007

Bajo, R., Maestú, F., Nevado, A., Sancho, M., Gutiérrez, R., Campo, P., et al. (2010). Functional connectivity in mild cognitive impairment during a memory task: implications for the disconnection hypothesis. J. Alzheimers Dis. 22, 183-193. doi: 10.3233/jad-2010-100177

Bendlin, B. B., Carlsson, C. M., Johnson, S. C., Zetterberg, H., Blennow, K., and Willette, A. (2012). CSF T-Tau/A $\beta 42$ predicts white matter microstructure in healthy adults at risk for Alzheimer's disease. PLoS One 7:e37720. doi: 10.1371/ journal.pone.0037720

Bullmore, E., and Sporns, O. (2009). Complex brain networks: graph theoretical analysis of structural and functional systems. Nat. Rev. Neurosci. 10, 186-198. doi: $10.1038 / \mathrm{nrn} 2575$

Cambria, E., and Huang, G.-B. (2013). Extreme learning machines. IEEE Intell. Syst. 28, 2-31.

Canu, E., McLaren, D. G., Fitzgerald, M. E., Bendlin, B. B., Zoccatelli, G., and Alessandrini, F. (2010). Microstructural diffusion changes are independent of macrostructural volume loss in moderate to severe Alzheimer's disease. J. Alzheimers Dis. 19, 963-976. doi: 10.3233/jad-2010-1295

Cao, J., Zhang, K., Luo, M., Yin, C., and Lai, X. (2016). Extreme learning machine and adaptive sparse representation for image classification. Neural Netw. 81, 91-102. doi: 10.1016/j.neunet.2016.06.001

Chao-Gan, Y., and Yu-Feng, Z. (2010). DPARSF: a MATLAB toolbox for "Pipeline" data analysis of resting-State fMRI. Front. Syst. Neurosci. 4:13. doi: 10.3389/ fnsys.2010.00013

Chen, Y. J., Deutsch, G., Satya, R., Liu, H. G., and Mountz, J. M. (2013). A semiquantitative method for correlating brain disease groups with normal controls using SPECT: Alzheimer's disease versus vascular dementia. Comput. Med. Imaging Graph. 37, 40-47. doi: 10.1016/j.compmedimag.2012.11.001

Cortes, C., and Vapnik, V. (1995). Support-vector networks. Mach. Learn. 20, 273-297.

de Vos, F., Koini, M., Schouten, T. M., Seiler, S., van der Grond, J., Lechner, A., et al. (2018). A comprehensive analysis of resting state fMRI measures to classify individual patients with Alzheimer's disease. Neuroimage 167, 62-72. doi: 10.1016/j.neuroimage.2017.11.025

Delbeuck, X., Collette, F., and Van der Linden, M. (2007). Is Alzheimer's disease a disconnection syndrome? Evidence from a crossmodal audio-visual illusory experiment. Neuropsychologia 45, 3315-3323.

Delbeuck, X., Van der Linden, M., and Collette, F. (2003). Alzheimer's disease as a disconnection syndrome? Neuropsychol. Rev. 13, 79-92.

Doan, N. T., Engvig, A., Persson, K., Alnæs, D., Kaufmann, T., Rokicki, J., et al. (2017). Dissociable diffusion MRI patterns of white matter microstructure and connectivity in Alzheimer's disease spectrum. Sci. Rep. 7:45131.

Du, L., and Shen, Y. D. (2015). "Unsupervised feature selection with adaptive structure learning," in Proceedings of the 21th ACM SIGKDD International Conference on Knowledge Discovery and Data Mining, 209-218.

Eavani, H., Satterthwaite, T. D., Gur, T. E., Gur, R. C., and Davatzikos, C. (2013). Unsupervised learning of functional network dynamics in resting state fMRI. Inf. Process Med. Imaging 23, 426-437. doi: 10.1007/978-3-642-388 68-2_36

Górriz, J. M., Segovia, F., Ramírez, J., Lassl, A., and Gonzalez, D. S. (2011). GMM based SPECT image classification for the diagnosis of Alzheimer's disease. Appl. Soft. Comput. 11, 2313-2325. doi: 10.1016/j.asoc.2010.08.012

Gray, K. R., Wolz, R., Heckemann, R. A., Aljabar, P., Hammers, A., and Rueckert, D. (2012). Multi-region analysis of longitudinal FDG-PET for the classification of Alzheimer's disease. Neuroimage 60, 221-229. doi: 10.1016/j.neuroimage. 2011.12.071
Grover, A., and Leskovec, J. (2016). "Node2Vec: scalable feature learning for networks," in Proceedings of the 22nd ACM SIGKDD International Conference on Knowledge Discovery and Data Mining, (New York, NY: ACM).

Guyon, I., Weston, J., Barnhill, S., and Vapnik, V. (2002). Gene selection for cancer classification using support vector machines. Mach. Learn. 46, $389-422$.

Hall, M. A. (2000). Correlation-based feature selection for discrete and numeric class machine learning. Proc. 17th Int'l Conf. Machine Learning 359-366.

Hanyu, H., Asano, T., Sakurai, H., Imon, Y., Iwamoto, T., Takasaki, M., et al. (1999). Diffusion-weighted and magnetization transfer imaging of the corpus callosum in Alzheimer's disease. J. Neurol. Sci. 167, 37-44. doi: 10.1016/s0022-510x(99) 00135-5

Hanyu, H., Sato, T., Hirao, K., Kanetaka, H., Iwamoto, T., and Koizumi, K. (2010). The progression of cognitive deterioration and regional cerebral blood flow patterns in Alzheimer's disease: a longitudinal SPECT study. J. Neurol. Sci. 290, 96-101. doi: 10.1016/j.jns.2009.10.022

Huang, G. B., Zhu, Q. Y., and Siew, C. K. (2006). Extreme learning machine: theory and applications. Neurocomputing 70, 489-501. doi: 10.1016/j.neucom.2005. 12.126

Khazaee, A., Ebrahimzadeh, A., and Babajani-Feremi, A. (2017). Classification of patients with $\mathrm{MCI}$ and $\mathrm{AD}$ from healthy controls using directed graph measures of resting-state fMRI. Behav. Brain Res. 322, 339-350. doi: 10.1016/j.bbr.2016. 06.043

Leonardi, N., Richiardi, J., Gschwind, M., Simioni, S., Annoni, J.-M., Schluep, M., et al. (2013). Principal components of functional connectivity: a new approach to study dynamic brain connectivity during rest. Neuroimage 83, 937-950. doi: 10.1016/j.neuroimage.2013.07.019

Liu, F., Zhou, L., Shen, C., and Yin, J. (2014). Multiple kernel learning in the primal for multi-modal Alzheimer's disease classification. IEEE J. Biomed. Health Inform. 18, 984-990. doi: 10.1109/jbhi.2013.2285378

Liu, Y., Yu, C., Zhang, X., Liu, J., Duan, Y., Alexander-Bloch, A. F., et al. (2014). Impaired long distance functional connectivity and weighted network architecture in Alzheimer's disease. Cereb. Cortex 24, 1422-1435. doi: 10.1093/ cercor/bhs 410

Ly, M., Canu, E., Xu, G., Oh, J., McLaren, D. G., Dowling, N. M., et al. (2014). Midlife measurements of white matter microstructure predict subsequent regional white matter atrophy in healthy adults. Hum. Brain Mapp. 35, 2044 2054. doi: 10.1002/hbm.22311

Masliah, E., Mallory, M., Hansen, L., DeTeresa, D., and Terry, R. (1993). Quantitative synaptic alterations in the human neocortex during normal aging. Neurology 43, 192-197. doi: 10.1212/wnl.43.1_part_ 1.192

Supekar, K., Menon, V., Rubin, D., Musen, M., and Greicius, M. D. (2008). Network analysis of intrinsic functional brain connectivity in Alzheimer's disease. PLoS Comput. Biol. 4:e1000100. doi: 10.1371/journal.pcbi.1000100

Peng, X., Lin, P., Zhang, T., and Wang, J. (2013). Extreme learning machine-based classification of ADHD using brain structural MRI data. PLoS One 8:e79476. doi: 10.1371/journal.pone.0079476

Pfefferbaum, A., Sullivan, E., Hedehus, M., Lim, K., Adalsteinsson, E., and Moseley, M. (2000). Age-related decline in brain white matter anisotropy measured with spatially corrected echo-planar diffusion tensor imaging. Magn. Reson. Med. 44, 259-268. doi: 10.1002/1522-2594(200008)44:2<259::aid-mrm13>3.0.co;2-6

Qureshi, M. N. I., Min, B., Jo, H. J., and Lee, B. (2016). Multiclass classification for the differential diagnosis on the ADHD subtypes using recursive feature elimination and hierarchical extreme learning machine:structural MRI Study. PLoS One 11:e0160697. doi: 10.1371/journal.pone.0160697

Rubinov, M., and Sporns, O. (2010). Complex network measures of brain connectivity: uses and interpretations. Neuroimage 52, 1059-1069. doi: 10. 1016/j.neuroimage.2009.10.003

Schmitter, D., Roche, A., Maréchal, B., Ribes, D., Abdulkadir, A., BachCuadra, M., et al. (2015). An evaluation of volume-based morphometry for prediction of mild cognitive impairment and Alzheimer's disease. NeuroImage 7, 7-17.

Sorg, C., Riedl, V., Mühlau, M., Calhoun, V. D., Eichele, T., Läer, L., et al. (2007). Selective changes of resting-state networks in individuals at risk for Alzheimer's disease. Proc. Nat. Acad. Sci. U.S.A. 104, 18760-18765. doi: 10.1073/pnas. 0708803104 
Sporns, O. (2011). The human connectome: a complex network. Ann. N. Y. Acad. Sci. 1224, 109-125. doi: 10.1111/j.1749-6632.2010. 05888.x

Stam, C. J., Jones, B. F., Manshandena, I., van Cappellen van Walsum, A. M., Montez, T., Verbunt, J. P. A., et al. (2006). Magnetoencephalographic evaluation of resting-state functional connectivity in Alzheimer's disease. Neuroimage 32, 1335-1344. doi: 10.1016/j.neuroimage.2006.05.033

Suk, H. I., Wee, C. Y., Lee, S. W., and Shen, D. (2016). State-space model with deep learning for functional dynamics estimation in restingstate fMRI. Neuroimage 129, 292-307. doi: 10.1016/j.neuroimage.2016. 01.005

Tibshirani, R. (1996). Regression shrinkage and selection via the lasso. J. R. Stat. Soc. Ser. B Stat. Methodol. 58, 267-288. doi: 10.1111/j.25176161.1996.tb02080.x

Wee, C. Y., Yap, P. T., Zhang, D., Wang, L., and Shen, D. (2014). Groupconstrained sparse fMRI connectivity modeling for mild cognitive impairment identification. Brain Struct. Funct. 219, 641-656. doi: 10.1007/s00429-0130524-8

Wu, X., Li, J., Ayutyanont, N., Protas, H., Jagust, W., Fleisher, A., et al. (2013). The receiver operational characteristic for binary classification with multiple indices and its application to the neuroimaging study of Alzheimer's disease. IEEE/ACM Trans. Comput. Biol. Bioinform. 10, 173-180. doi: 10.1109/tcbb. 2012.141
Zeng, H., and Cheung, Y. (2011). Feature selection and kernel learning for local learning-based clustering. IEEE Transactions on PAMI 33, 1532-1547. doi: 10.1109/TPAMI.2010.215

Zhang, W., Shen, H., Ji, Z., Meng, G., and Wang, B. (2015). "Identification of mild cognitive impairment using extreme learning machines model," intelligent computing theories and methodologies," in Proceedings of the 11th International Conference, ICIC 2015, Fuzhou.

Zhou, J., Greicius, M. D., Gennatas, E. D., Growdon, M. E., Jang, J. Y., Rabinovici, G. D., et al. (2010). Divergent network connectivity changes in behavioural variant frontotemporal dementia and Alzheimer's disease. Brain 133, 13521367. doi: 10.1093/brain/awq075

Conflict of Interest: The authors declare that the research was conducted in the absence of any commercial or financial relationships that could be construed as a potential conflict of interest.

Copyright (C) 2021 Lama and Kwon. This is an open-access article distributed under the terms of the Creative Commons Attribution License (CC BY). The use, distribution or reproduction in other forums is permitted, provided the original author(s) and the copyright owner(s) are credited and that the original publication in this journal is cited, in accordance with accepted academic practice. No use, distribution or reproduction is permitted which does not comply with these terms. 